\title{
Sensor Data Validation and Reconstruction in Water Networks: a Methodology and Software Implementation
}

\author{
Diego García, Joseba Quevedo, Vicenç Puig, and Miquel Àngel Cugueró \\ Intelligent/Advanced Control Systems (SIC/SAC), \\ Universitat Politècnica de Catalunya (UPC), \\ Terrassa (Barcelona), Catalunya, Spain \\ \{diego.garcia, joseba.quevedo, vicenc.puig, miquel.angel.cuguero\}@upc.edu \\ http://sac.upc.edu
}

\begin{abstract}
In this paper, a data validation and reconstruction methodology that can be applied to the sensors used for real-time monitoring in water networks is presented. On the one hand, a validation approach based on quality levels is described to detect potential invalid and missing data. On the other hand, the reconstruction strategy is based on a set of temporal and spatial models used to estimate missing/invalid data with the model estimation providing the best fit. A software tool implementing the proposed data validation and reconstruction methodology is also presented. Finally, results obtained applying the proposed methodology on raw data of flow meters gathered from a real water network are also included to illustrate the performance of the proposed approach.
\end{abstract}

Keywords: data validation, data reconstruction, time series, data mining

\section{Introduction}

Water networks are Critical Infrastructure Systems (CIS) geographically distributed and decentralized, involving a large number of sensors for real-time monitoring and efficient and safe operation of these networks.

However, in real water network operation, problems affecting the communication system between sensors and data loggers, or in the telecontrol system itself, often arise generating missing data during certain periods of time. The data recorded by these sensors are sometimes uncorrelated so data coming from healthy sensors cannot be used to replace unhealthy sensor missing data, which therefore must be replaced by a set of estimated data. Another common problem in such systems is the lack of sensor reliability (due to e.g. offset, drift or breakdowns) producing false flow data readings. These unreliable data must also be detected and replaced by estimated data, since sensor data are used for several network water management tasks, namely: planning, investment plans, maintenance operations, billing/consumer services and operational control. Furthermore, SCADA and telemetry systems generate extremely heterogeneous data. 
Thus, working on raw data involves a lot of $a d$-hoc scripting to prepare them to be treated and processed, when actually the efforts should be focused on manipulating the data to be used for the efficient network management. As a result, a fragmented and heterogeneous data repository is obtained, making it tedious to work with stored raw sensor data and slowing down the overall data treatment process. Improving this effect has motivated the work presented here.

According to the nature of the available knowledge, different kinds of data validation approaches may be considered, with varying degrees of sophistication. In general, one may distinguish between elementary signal-based ("low-level") methods and model-based ("higher level") methods (see e.g. [7]). Elementary signal based methods use simple heuristics and limited statistical information of a given sensor [2]. Typically, these methods are based on validating either signal values or signal variations. On the one hand, in the signal value-based approach data are assessed as valid or invalid according to two different thresholds (high and low) so data is assumed to be invalid when lying outside these threshold values. On the other hand, methods based on signal variations look for strong variations (peaks in the curve) as well as lack of variations (flat curve). Modelbased methods rely on the use of models to check the consistency of sensor data [8]. This consistency check is based on computing the difference between the predicted value from the model and the real value measured by the sensors. Then, this difference (known as residual) is compared with a threshold value (zero in the ideal case). When the residual is bigger than the corresponding threshold, a fault is assumed in the sensor; otherwise, the sensor is assumed to work properly. Moreover, the information of all the available residuals and models allows performing fault isolation in order to discover the faulty sensor. Models are usually derived using either multivariate procedures exploiting the correlation or the analytical relations between several quantities obtained using first principles, sometimes measured at different times ("temporal redundancy") and/or locations ("spatial redundancy"). A methodology to validate and reconstruct missing/invalid sensor data, previously introduced in [6], is applied here to data coming from flow meters in the Catalonia water transport network. In this paper, the main contribution is a software tool implementing this methodology that is able to properly handle raw sensor data (including storage, querying and visualization).

The structure of the paper is as follows: Section 2 introduces the proposed sensor validation and reconstruction methodology. Section 3 describes a software tool that implements the proposed methodology. Section 4 illustrates the application of the proposed methodology and the software tool to a real water network. Finally, Section 5 draws the main conclusions achieved.

\section{Methodology}

The methodology presented here, depicted in Fig. 1, is designed to validate and reconstruct invalid and missing sensor data. This methodology is detailed in the next sections. 


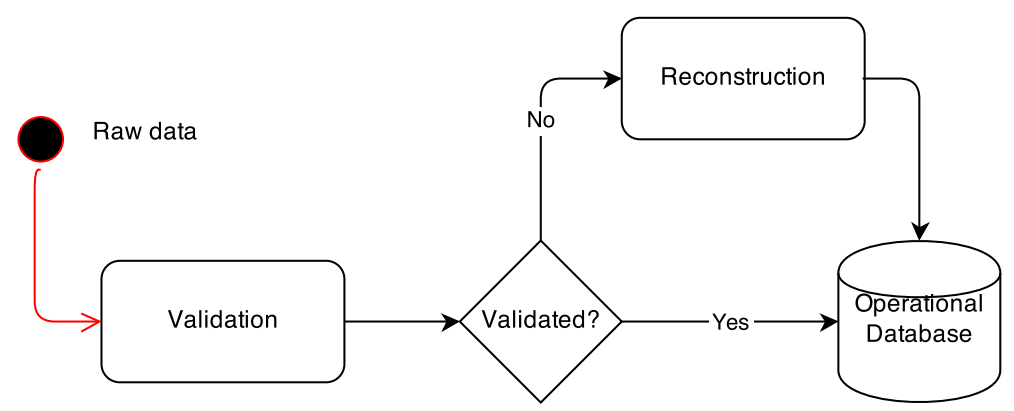

Fig. 1. Validation and reconstruction data flow diagram.

\subsection{Sensor Data Validation}

The sensor data validation methodology is inspired in the Spanish AENOR-UNE norm 500540 [7]. The methodology applies a set of consecutive validation tests to a given dataset (Fig. 2) to finally assign a certain quality level depending on the tests passed. The six different quality levels are the following:

- Level 0: This level allows to detect data acquisition and communication errors.

- Level 1: Any sensor has an operational measurement interval. Any value above or beyond this interval is invalidated by this level.

- Level 2: The trend level takes into account the data changes over time. This allows to detect unexpected changes in the data, e.g. a tank level sensor cannot decrease or increase more than a certain height in an hour due to configuration's limitations (in this particular case, maximum input and maximum output flows).

- Level 3: This level allows to check the variables in a given unit, e.g. a flow meter cannot measure a non-zero value if the valve located at the same pipe is totally closed.

- Level 4: This level evaluates the sensor's measurements against an estimated data given by a time series model based on historical data [4].

- Level 5: This level checks the correlation between different neighboring sensors [5], e.g. two flow sensors located at the same pipe without any element in the middle (e.g. node, tank) must measure.

\subsection{Sensor Data Reconstruction}

Time Series Models (TSM) and Spatial Models (SM) are used to reconstruct missing and invalid data. The former are based on time series' features such as seasonality, trend and periodicity, in order to estimate future values based on hystorical behavior. Two TSMs are used in the proposed methodology/tool: an exponential smoothing Holt-Winters model and a generalized ARIMA model. 


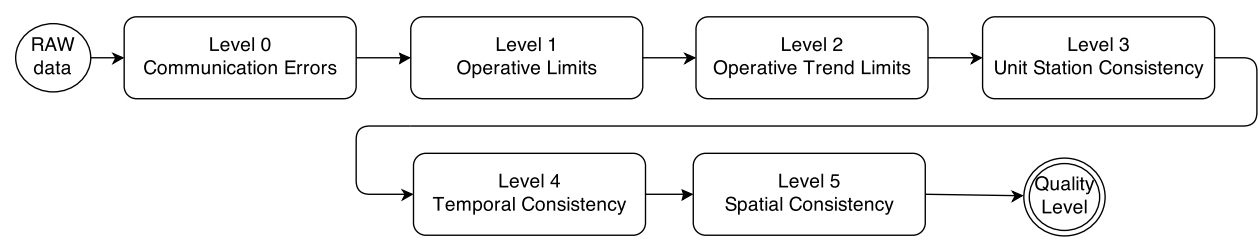

Fig. 2. Validation data flow diagram.

On the one hand, a spread method for time series modelling because of its simplicity and performance is the Holt-Winters (HW) approach $[3,9]$. There are various approaches for this method, e.g. additive or damped trend, additive or multiplicative seasonality, single or multiple seasonality. Here, good performance is achieved with the additive single seasonality approach, with estimated value for a forecasting horizon $\ell$ as follows

$$
\hat{y}_{T S 1}(k)=\bar{R}(k-\ell)+\ell \bar{G}(k-\ell)+\bar{S}(k-L)
$$

where $\bar{R}$ is the level component

$$
\begin{aligned}
\bar{R}(k-\ell)= & \alpha(y(k-\ell)-\bar{S}(k-L-\ell))+(1-\alpha)(\bar{R}(k-\ell-1) \\
& +\bar{G}(k-\ell-1))
\end{aligned}
$$

$\bar{G}$ is the trend component

$$
\bar{G}(k-\ell)=\beta(\bar{R}(k-\ell)-\bar{R}(k-\ell-1))+(1-\beta) \bar{G}(k-\ell-1)
$$

$\bar{S}$ is the seasonal component

$$
\bar{S}(k-\ell)=\gamma(y(k-\ell)-\bar{R}(k-\ell))+(1-\gamma) \bar{S}(k-\ell-L) .
$$

and $L$ is the season (daily here) periodicity, $\alpha, \beta$ and $\gamma$ are the HW parameters (level, trend and season smoothing factors, respectively), $y$ is the measured value (flow meter raw data here) and $\hat{y}_{T S 1}(k)$ is the TS model forecasted value at time instant $k$. The parameters $\alpha, \beta$ and $\gamma$ are inside the interval $[0,1]$ and can be estimated from historical data using the least-squares approach.

On the other hand, the generalized, well known and useful ARIMA model for time series $[1,7]$ is described for hourly-sampled data by the following difference equation, which describes a periodicity of one day (24 hours)

$$
\hat{y}_{T S 2}(k)=-a_{1} y(k-1)-a_{2} y(k-2)-a_{3} y(k-3)-\ldots-a_{24} y(k-24),
$$

based on the following three components, first

$$
y_{\text {int }}(k)=y(k)-y(k-1)
$$

takes into account the level and the trend, second 


$$
y_{o s c}(k)=y_{i n t}(k)-2 \cos \left(\frac{2 \pi}{24}\right) y_{i n t}(k-1) y(k)+y_{i n t}(k-2)
$$

considers the daily seasonality, and finally

$$
\hat{y}_{T S 2}(k)=-a_{1} y_{\text {osc }}(k-1)-a_{2} y_{\text {osc }}(k-2)-\ldots-a_{21} y(k-21)
$$

includes an autoregressive component to consider the influence of previous flow values within a day.

Alternatively, SM are linear regression models relating different spatially dependant measurements in the system, stated as follows

$$
\hat{y}_{s}(k)=a y_{f}(k)+b .
$$

where $a$ and $b$ are the parameters of the model to be calibrated. For instance, two flow meters installed in the same pipe and separated by a certain distance should measure a similar flow value if there is no element e.g. reservoir or node located between them i.e. $a \approx 1, b \approx 0$. Due to the network's configuration maybe a SM is not available, thus only temporal models could be used.

Furthermore, the Mean Squared Error (MSE) defined as

$$
M S E(k)=\frac{1}{m} \sum_{j=k-m}^{k}(y(j)-\hat{y}(j))^{2}
$$

is the model accuracy measure. The model with the lowest MSE over the $m$ estimations previous to $k$ is the selected candidate to estimate the invalid $/ \mathrm{missing}$ $k$-sample.

\section{Software Implementation}

The architecture of the software tool implemented is depicted in Fig. 3. There are two main components: the Data Management Web application and the Validation and Reconstruction tool ${ }^{1}$.

The Data Management component is a web application. Thus, it allows the access to the data from everywhere. The Validation and Reconstruction component is implemented in Matlab.

\subsection{Data Management Web Application}

This module provides a user-friendly tool allowing to import and export data so that stored data is available to all the registered users. In order to focus the efforts on the data intrinsic values and not on how to access them, the tool provides three main services:

\footnotetext{
${ }^{1}$ Both software tools are proprietary software.
} 


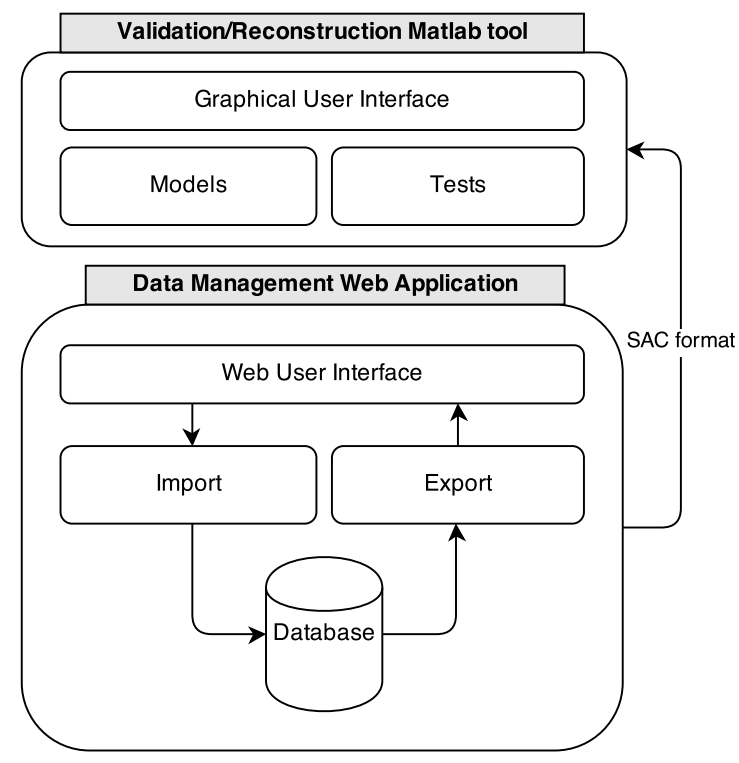

Fig. 3. Software architecture diagram.

- Data importation from different sources (e.g. CSV, Excel, Access).

- Data exportation of a determined time period in three different formats:

CSV, Excel and SAC format ${ }^{2}$.

- Data visualization.

This data management component is implemented using Django ${ }^{3}$ web framework, providing a Web User Interface implemented in HTML and JavaScript in order to interact with the Import and Export Python modules (Fig. 4). The Import and Export modules handle the operations of saving and querying data against the Postgresql Database server.

\subsection{Validation and Reconstruction Matlab Tool}

The Validation and Reconstruction methodologies (described in Sections 2.1 and 2.2) are implemented in Matlab, a widely used numerical computing and programming platform in many research institutions and industrial enterprises, which makes it a convenient prototyping and developement framework.

This tool applies the presented methodologies to the data in three different stages: Calibration, Validation and Reconstruction. Fig. 5 shows the data flow between these stages. Calibration stage uses historic data to learn and estimate the parameters required by the tests and models described in Sections 2.1 and

\footnotetext{
${ }^{2}$ SAC format is a binary Mat-file containing a defined data structure.

${ }^{3}$ Django is a free open source web framework. Its primary goal is to facilitate the creation of complex, database-driven websites.
} 


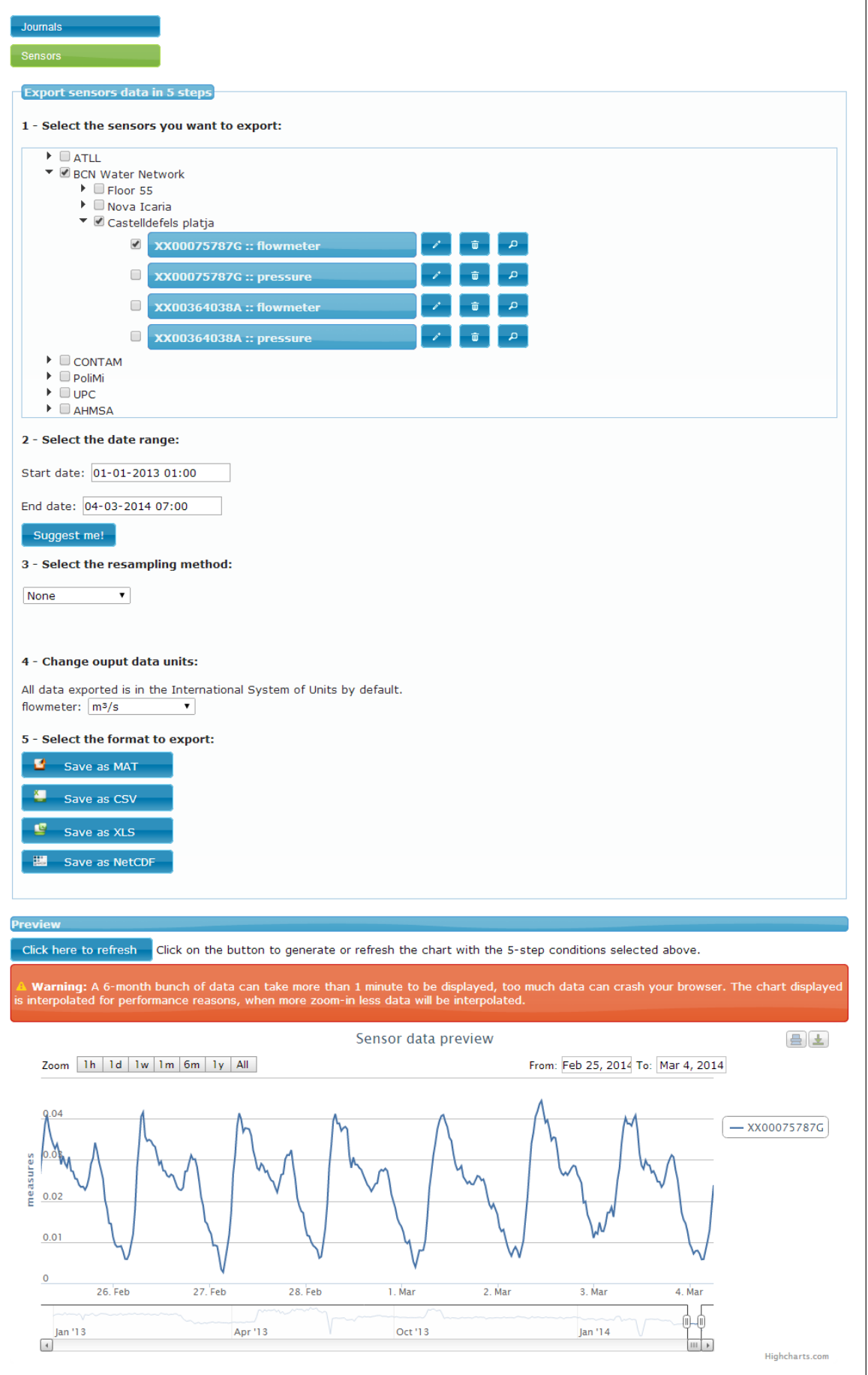

Fig. 4. Data Management Web Application screenshot. 
2.2. Calibration stage runs only once, in order to initialize the paramaters required by the following stages. Validation stage implements the methodology of Section 2.1. Finally, Reconstruction stage implements the methodology described in Section 2.2.

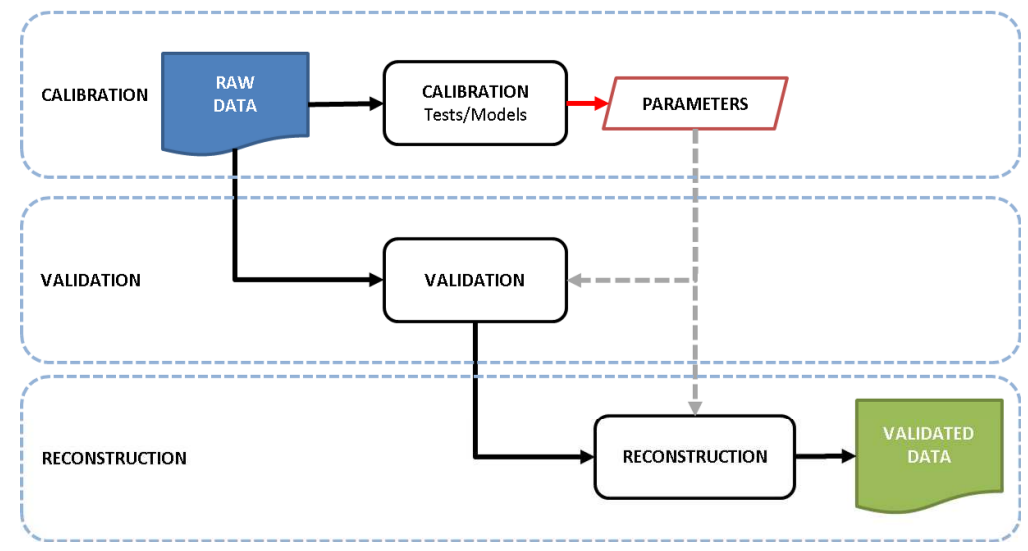

Fig. 5. Validation and Reconstruction data flow diagram.

This tool includes a Graphical User Interface (GUI in Fig. 6) to select the input and output parameters.

\section{Results}

In this section, some results obtained from the Validation and Reconstruction Matlab Tool applying the methodology described here to data coming from a real water network, are presented. The dataset is composed by hourly sampled data coming from two different flow meters ("A" and "B") installed in the Catalonia water transport network, managed by Aigües Ter Llobregat (ATLL) company. Fig. 7 shows four consecutive days of measurements gathered by the flow meter "A". As it may be seen, two peaks, at 12:00 and 18:00, in the first day that they do not fit the daily flow pattern shown in the following days. Thus, these two peaks are invalidated by the ARIMA model. Finally, these two samples are reconstructed by the model having the lowest MSE, which is also the ARIMA model.

Another scenario, corresponding to the flow meter "B", is shown in Fig. 8. Similarly as in the first scenario, some values in the fifth day do not fit the 


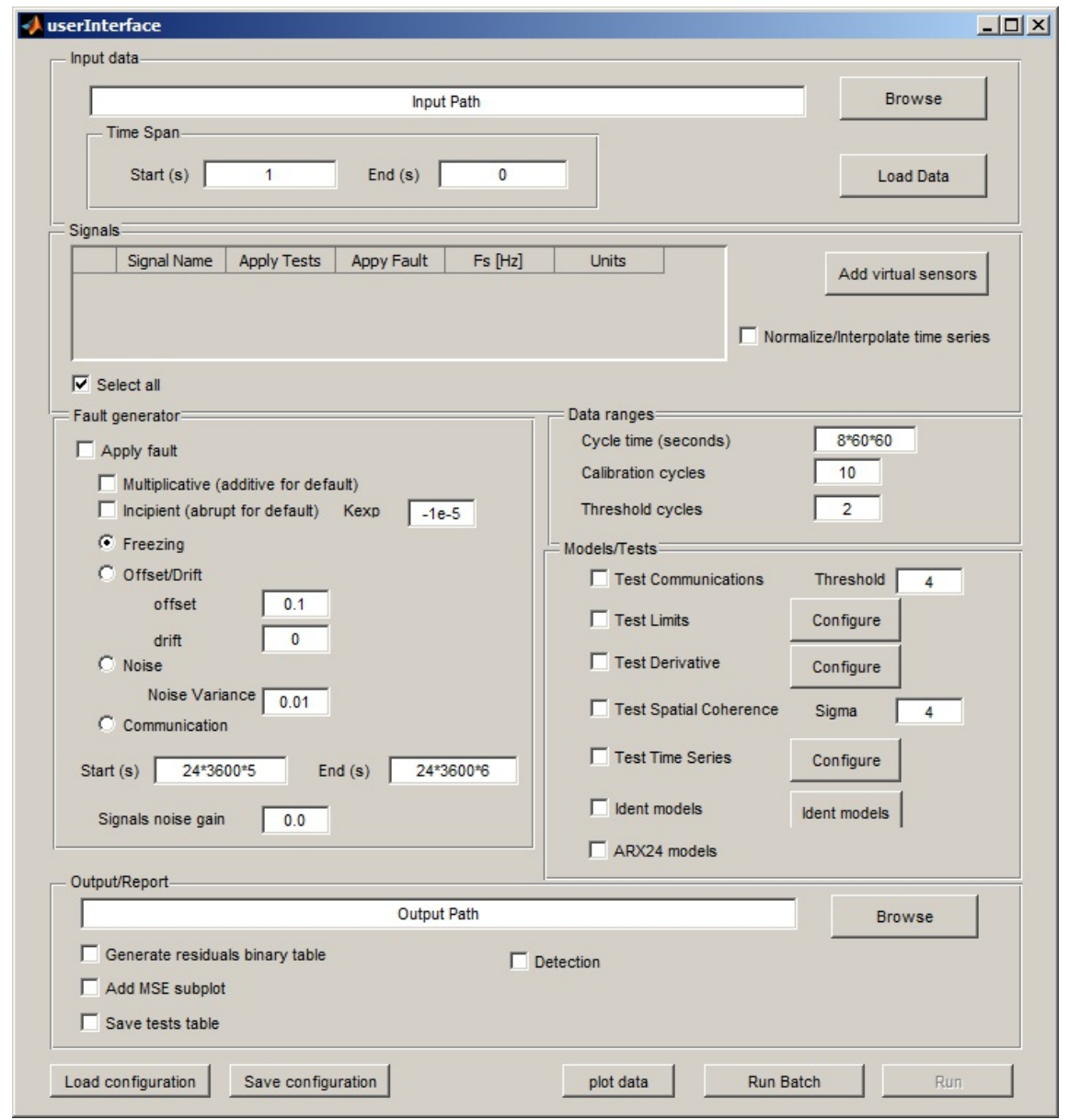

Fig. 6. Validation and Reconstruction Matlab Tool. 

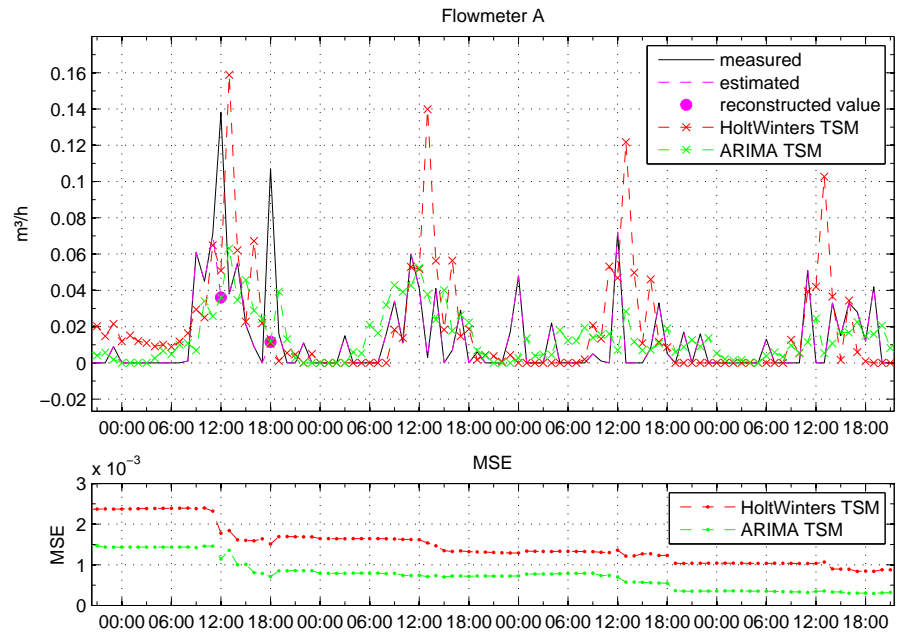

Validation

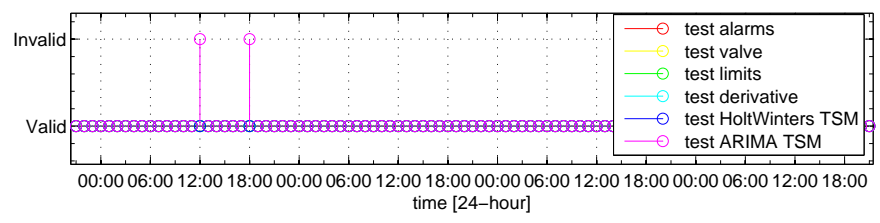

Fig. 7. Results of the validation and reconstruction methodology on the flow meter "A". 
daily flow pattern. Thus, these values are invalidated and reconstructed by the ARIMA model, which again is the one having lowest MSE.
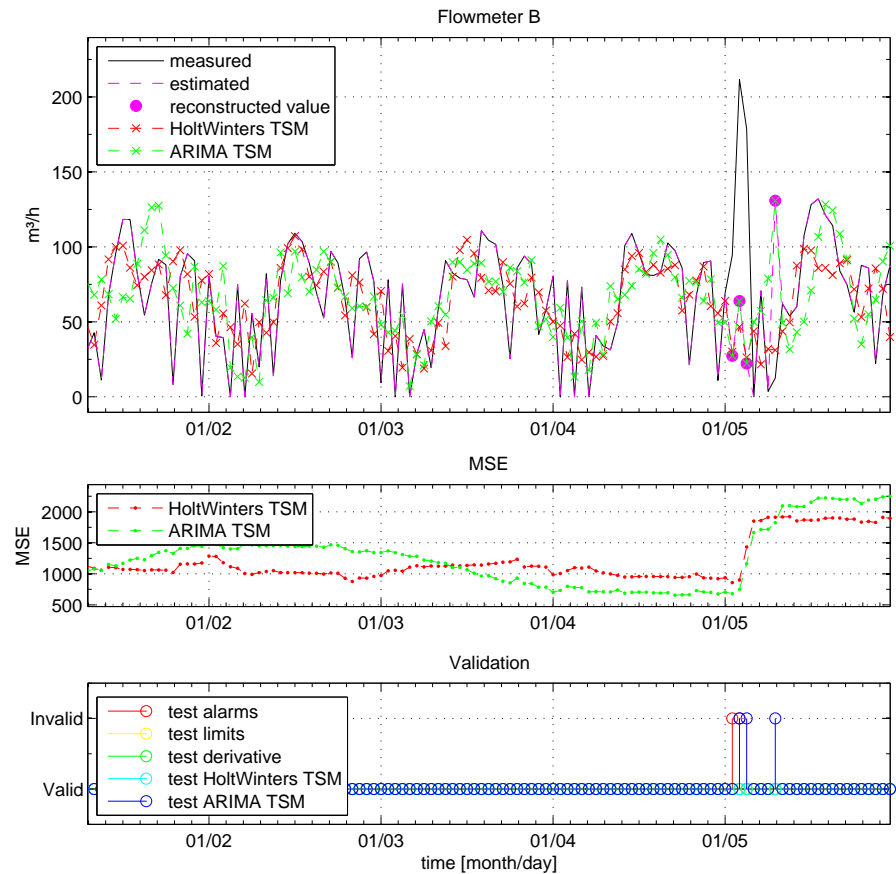

Fig. 8. Results of the validation and reconstruction methodology on the flow meter "B".

\section{Conclusions}

In this paper, a data validation and reconstruction framework is introduced to overcome the sensor problems arising in CIS, such as water networks. A validation strategy based on a set of data quality tests allows to detect suspicious erroneous data. Then, a reconstruction scheme is defined using Temporal and Spatial Models to provide an estimation based on the model having best fit. In addition, a software tool is described to provide a homogeneous and accessible database by a user-friendly interface, to easily apply the methodology presented here. Finally, some results obtained using data from a real network are presented using the software described, proving the ability of the methodology to detect and reconstruct anomalous data. 


\section{Acknowledgements}

This work is partially supported by CICYT SHERECS DPI-2011-26243 of the Spanish Ministry of Education, by EFFINET grant FP7-ICT-2012-318556 of the European Commission and by AGAUR Doctorat Industrial 2013-DI-041. The authors also wish to thank the support received by the company ATLL in the development of this work.

\section{References}

1. Blanch, J., Puig, V., Saludes, J., Quevedo, J.: Arima models for data consistency of flowmeters in water distribution networks. In: 7th IFAC Symposium on Fault De-tection, Supervision and Safety of Technical Processes. pp. 480-485 (2009)

2. Burnell, D.: Auto-validation of district meter data. In: CCWI '03 Advances in Water Supply Management. London (2003)

3. Makridakis, S., Wheelwright, S., Hyndman, R.: Forecasting methods and applications. John Wiley \& Sons (1998)

4. Quevedo, J., Blanch, J., Puig, V., Saludes, J., Espin, S., Roquet, J.: Methodology of a data validation and reconstructions tool to improve the reliability of the water network supervision. In: International Conference of IWA Water Loss. Sao Paolo, Brazil (2010)

5. Quevedo, J., Blanch, J., Puig, V., Saludes, J., Roquet, J., Espin, S.: Methodology to determine the drinking water transport network efficiency based on interval computation of annual performance. Revista Automática e Instrumentación 408, 44-49 (2009)

6. Quevedo, J., Chen, H., Cugueró, M.A., Tino, P., Puig, V., García, D., Sarrate, R., Yao, X.: Combining learning in model space fault diagnosis with data validation/reconstruction: Application to the barcelona water network. Engineering Applications of Artificial Intelligence 30(0), 18-29 (Apr 2014), http://www . sciencedirect.com/science/article/pii/S0952197614000153

7. Quevedo, J., Puig, V., Cembrano, G., Blanch, J., Aguilar, J., Saporta, D., Benito, G., Hedo, M., Molina, A.: Validation and reconstruction of flow meter data in the barcelona water distribution network. Control Engineering Practice 18(6), 640-651 (Jun 2010)

8. Tsang, K.: Sensor data validation using gray models. ISA Transactions 42, 9-17 (2003)

9. Winters, P.R.: Forecasting sales by exponentially weighted moving averages. Management Science 6(52), 324-342 (1960) 